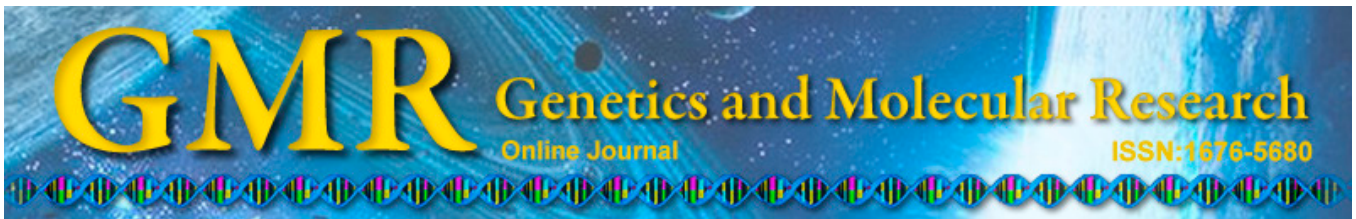

\title{
Advanced oxidation protein products as a biomarker of cutaneous lupus erythematosus complicated by nephritis: a case-control study
}

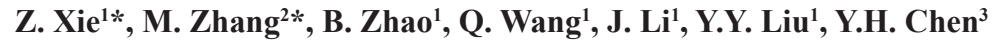 \\ ${ }^{1}$ Department of Dermatology, \\ Sichuan Academy of Medical Sciences \& Sichuan Provincial People's Hospital, \\ Chengdu, China \\ ${ }^{2}$ Department of Gastroenterology, the Sixth Affiliated Hospital, \\ Sun Yat-Sen University, Guangzhou, China \\ ${ }^{3}$ Division of Nephrology, Guangdong General Hospital, \\ Guangdong Academy of Medical Sciences, Guangzhou, China \\ *These authors contributed equally to this study. \\ Corresponding author: Y.H. Chen \\ E-mail: xiezhencn1234@163.com
}

Genet. Mol. Res. 13 (4): 9213-9219 (2014)

Received October 16, 2013

Accepted January 13, 2014

Published November 7, 2014

DOI http://dx.doi.org/10.4238/2014.November.7.8

\begin{abstract}
Oxidative stress is involved in the pathogenesis of lupus nephritis (LN). The current study investigated the significance of advanced oxidation protein products (AOPPs) as a biomarker of LN in patients with cutaneous lupus erythematosus. Ninety-two patients who initially presented with systemic lupus erythematosus were divided into the $\mathrm{LN}-$ and $\mathrm{LN}+$ groups. Serum AOPP levels were determined, and the association between AOPP levels and LN was investigated in a case-control study. In the $\mathrm{LN}+$ group, patients with higher AOPP levels exhibited higher levels of dsDNA and proteinuria but lower levels of eGFR and complement $\mathrm{C} 3$ compared to those in patients with lower AOPP levels. A multivariable logistic regression model showed that the AOPP level was an independent risk factor for
\end{abstract}


LN. The risk of nephritis specifically increased $24 \%$ for each $10 \mu \mathrm{M}$ increase in AOPP (95\% confidence interval, 1.166-1.915, $\mathrm{P}=0.030$ ). In contrast, neither elevated dsDNA level nor decreased complement C3 level was an independent risk factor for LN. Higher serum AOPP levels were associated with an increased risk of LN. Therefore, future studies are warranted to determine the potential clinical value of this novel biomarker.

Key words: Advanced oxidation protein products; Lupus nephritis; Cutaneous lupus erythematosus; Biomarker; Case-control study; Prognosis

\section{INTRODUCTION}

Systemic lupus erythematosus (SLE) is a primary autoimmune disease. It is not uncommon in Asian countries including China (Mok, 2011). SLE has complex pathogenic mechanisms and can cause injuries to multiple organs. In China, the kidneys are the most susceptible organs to SLE development after the skin. Because the treatment protocols and prognoses for different types of SLE with or without lupus nephritis (LN) can vary greatly, the ability to differentiate between cutaneous lupus erythematosus and LN is clinically important (Fismen et al., 2007).

Neutrophil-mediated oxidative stress is an important mechanism of LN (Knight and Kaplan, 2012). Oxidation proteins contain double-tyrosine structures. Meanwhile, advanced oxidation protein products (AOPPs) are generated by myeloperoxidase in neutrophils because of oxidative stress (Capeillere-Blandin et al., 2006). The double-tyrosine structure of AOPPs has a special absorbance feature at $340 \mathrm{~nm}$. Taking advantage of this absorbance property, Witko-Sarsat et al. (1996) developed an economical and convenient spectrophotometric method to detect AOPPs that has been used extensively in recent years. Thus, AOPPs are considered a biological marker that reflects inflammation-associated oxidative stress (Witko-Sarsat et al., 1996). AOPPs can activate inflammatory cells (Witko-Sarsat et al., 1998) and contribute to the progression of renal failure (Li et al., 2007). AOPPs have been used as markers of autoimmune diseases of the skin and kidneys (Descamps-Latscha et al., 2004; Yazici et al., 2004; Li et al., 2007; Servettaz et al., 2007). Furthermore, a subset of SLE patients present with increased serum levels of AOPPs (Lozovoy et al., 2011; Hanasand et al., 2012).

Accordingly, the present case-control study aimed to determine whether the AOPP level can be used as a biomarker to predict LN in SLE patients who initially present with cutaneous lupus erythematosus.

\section{MATERIAL AND METHODS}

\section{Patient selection}

SLE patients hospitalized from January 2010 to December 2012 for skin manifestations in the Department of Dermatology of the Sichuan Provincial People's Hospital were analyzed retrospectively. These patients' manifestations of cutaneous lupus erythematosus were aligned with the classification criteria for SLE-characteristic skin lesions established 
by the Systemic Lupus International Collaborating Clinics (Petri and Magder, 2004). These inpatients were studied and divided into the LN- and LN+ groups on the basis of their renal complications. In addition, patients were included if they were followed up for at least 6 months and their serum samples from their initial admissions were available. The LN patients who were followed were placed in the $\mathrm{LN}+$ group even if they did not have renal damage on admission. The exclusion criteria were as follows: 1) involvement of organs besides the kidneys; 2) a clinical manifestation or examination outcome of infection during the first 4 weeks of the investigation; and 3) diabetes mellitus. A total of 140 patients were originally enrolled, but 24 were excluded because of a lack of available serum samples. Among the remaining 116 patients, 106 were successfully followed for 6 months. During the followup, extrarenal organ damage, concurrent infections, and diabetes occurred in 3, 5, and 6 patients, respectively; these patients were excluded from the analysis. Finally, 92 patients were analyzed, including 56 and 36 patients in the LN- and LN+ groups, respectively. In the $\mathrm{LN}+$ group, 30 patients underwent percutaneous renal needle biopsies. Meanwhile, the other 6 patients did not consent to renal biopsy. According to the LN pathological classifications proposed by the World Health Organization, 6, 21, 2, 1 patients were type III, IV, V, and IV $+\mathrm{V}$, respectively.

This study was conducted in accordance with the Declaration of Helsinki and was approved by the institutional ethics committee of Sichuan Provincial People's Hospital, Chengdu, China. Informed consent was obtained from all participants prior to participation.

\section{Clinical indices}

The patients' demographic data, clinical manifestations (including dental ulcers, arthritis, and serositis manifestations), hematological abnormalities, renal function, immunological abnormalities, and inflammatory indices upon hospitalization were collected. SLE-associated hematological damage was evaluated according to the criteria proposed by the American College of Rheumatology, including leukocyte count $<4.0 \times 10^{9} / \mathrm{L}$, hemolytic anemia, and platelet count $<100 \times 10^{9} / \mathrm{L}$ (Bombardier et al., 1992). The estimated glomerular filtration rate (eGFR) was calculated on the basis of the EPI formula to reflect renal function (Levey et al., 2009). Complement $\mathrm{C} 3<0.8 \mathrm{~g} / \mathrm{L}$ was defined as hypocomplementemia, and dsDNA $>100 \mathrm{IU} / \mathrm{mL}$ was defined as dsDNA positive on the basis of the laboratory index used at the Sichuan Provincial People's Hospital. SLE activity was evaluated on the basis of the SLE disease activity index (SLEDAI, Bombardier et al., 1992).

\section{Serum AOPP detection}

On admission, serum samples were collected for AOPP detection. Serum samples $(200 \mu \mathrm{L})$ or chloramine-T standards (Sigma, USA) were added to a 96-well plate (Corning Costar, Corning, USA). Each serum sample was mixed with $20 \mu \mathrm{L}$ acetic acid, and the chloramine-T standards were mixed with $30 \mu \mathrm{L}$ potassium iodide-acetic acid reaction solution $(1: 2 \mathrm{v} / \mathrm{v})$. The absorbance (A) was immediately read at $340 \mathrm{~nm}$ (Thermo Multiskan MK3, Finland) (Chen et al., 2011). All samples were measured in triplicate, and the detection was completed within 3 min of sample application. 


\section{Statistical analysis}

The data were analyzed using SPSS version 13.0 (SPSS Inc., USA). Two independentsample $t$-tests were used to analyze numerical variables, and the $\chi^{2}$ test was used to analyze nominal variables for intergroup comparisons. Pearson's correlation analysis was performed to determine the correlations between 2 variables. A multivariable binary logistic regression model was used to analyze the risk factors contributing to nephritis. In the intergroup comparison, variables $(\mathrm{P}<0.10)$ were introduced into the multivariate logistic regression model to analyze the risk of LN. Odds ratios and $95 \%$ confidence intervals (CIs) were used to evaluate the correlations between the risk factors and subsequent disease risk. Some of the variables were transformed appropriately according to their clinical significance. The level of statistical significance was set at $\mathrm{P}<0.05$, and all tests were 2-sided.

\section{RESULTS}

\section{Patient characteristics}

No significant differences were observed between the 2 groups with respect to age, gender, or clinical signs and symptoms. However, the LN+ group exhibited higher dsDNA levels, SLEDAI scores, and serum AOPP levels but lower eGFR compared to those in the LN- group (Table 1).

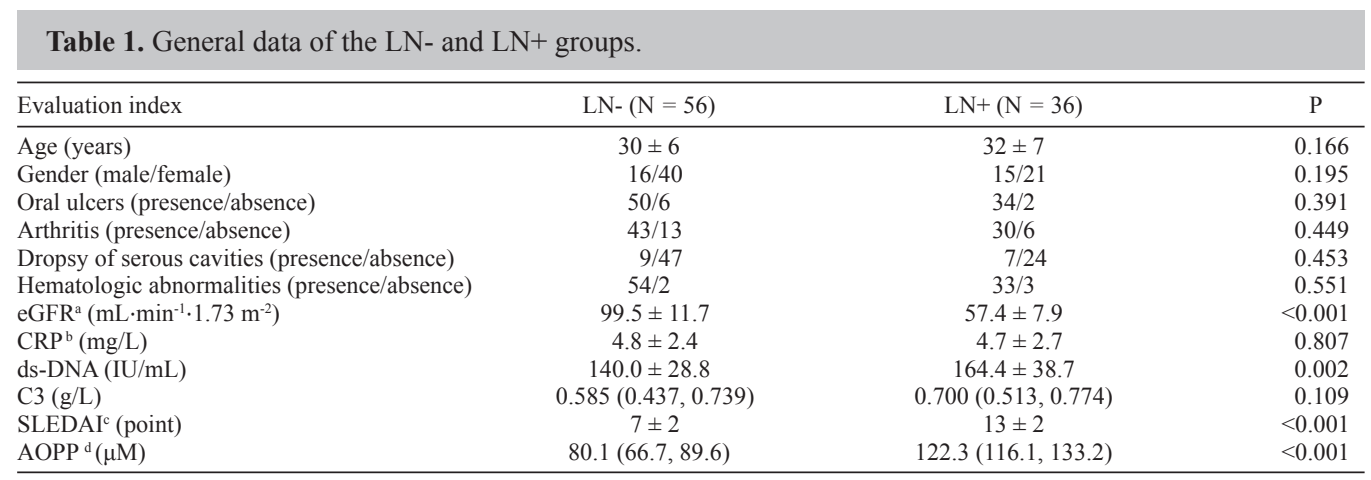

Continuous variables are reported as mean values \pm SD or medians (25th, 75th percentile) as appropriate and categorical data are reported as number of cases (percentage). ${ }^{\mathrm{a} E s t i m a t e d ~ g l o m e r u l a r ~ f i l t r a t i o n ~ r a t e ; ~}{ }^{\mathrm{b}} \mathrm{C}-$ reactive protein; ${ }^{\mathrm{c} S y s t e m i c ~ l u p u s ~ e r y t h e m a t o s u s ~ d i s e a s e ~ a c t i v i t y ~ i n d e x ; ~}{ }^{\mathrm{d} A d v a n c e d}$ oxidation protein products.

\section{Correlation between AOPP and LN}

SLEDAI scores (1 point per increase), dsDNA levels (10 IU/mL per increase), and AOPP levels $(10 \mu \mathrm{M}$ per increase) were introduced into the logistic regression model. AOPP levels were a significant independent risk factor for LN in SLE patients initially presenting with cutaneous lupus erythematosus. Specifically, each $10-\mu \mathrm{M}$ increase in AOPP level increased the risk of LN $24 \%(95 \% \mathrm{CI}, 1.166-1.915, \mathrm{P}=0.030)$.

\section{LN+ subgroup analysis}

The 36 patients in the LN+ group was subdivided into the high and low AOPP sub- 
groups according to the AOPP median of $122.3 \mu \mathrm{M}$. The low AOPP subgroup exhibited higher dsDNA levels, SLEDAI scores, and serum AOPP levels but exhibited lower eGFR compared to those in the high AOPP subgroup (Table 2). Serum AOPP levels were positively correlated with SLEDAI scores and proteinuria levels, and negatively correlated with eGFR and dsDNA levels (Figure 1).

Table 2. General data of the 36 patients in the LN+ subgroup according to AOPP levels.

\begin{tabular}{|c|c|c|c|}
\hline Evaluation index & Low AOPP $(\mathrm{N}=18)$ & $\operatorname{High}$ AOPP $(\mathrm{N}=18)$ & $\mathrm{P}$ \\
\hline Age (years) & $30 \pm 6$ & $31 \pm 6$ & 0.761 \\
\hline Gender (male/female) & $6 / 12$ & 9/9 & 0.310 \\
\hline $\mathrm{eGFR}^{\mathrm{a}}\left(\mathrm{mL} \cdot \mathrm{min}^{-1} \cdot 1.73 \mathrm{~m}^{-2}\right)$ & $62.4 \pm 7.0$ & $52.3 \pm 5.0$ & $<0.001$ \\
\hline Proteinuria $(\mathrm{g} / 24 \mathrm{~h})$ & $2.8 \pm 1.2$ & $3.5 \pm 0.8$ & 0.031 \\
\hline $\mathrm{CRP}^{\mathrm{b}}(\mathrm{mg} / \mathrm{L})$ & $5.4 \pm 2.4$ & $4.1 \pm 3.0$ & 0.164 \\
\hline ds-DNA (IU/mL) & $151.7 \pm 34.1$ & $172.2 \pm 39.7$ & 0.047 \\
\hline $\mathrm{C} 3(\mathrm{mg} / \mathrm{L})$ & $0.700(0.552,0.751)$ & $0.493(0.421,0.731)$ & 0.152 \\
\hline SLEDAI $^{\mathrm{c}}$ (point) & $11.7 \pm 2.2$ & $13.9 \pm 2.1$ & 0.004 \\
\hline
\end{tabular}

${ }^{\mathrm{a}}$ Estimated glomerular filtration rate; ${ }^{\mathrm{b}} \mathrm{C}$-reactive protein; ${ }^{\mathrm{c}}$ Systemic lupus erythematosus disease activity index. Continuous variables are reported as mean values $\pm \mathrm{SD}$ or medians (25th, 75th percentile) as appropriate and categorical data as number of cases (percentage). The subgroups were subdivided according to the AOPP median of $122.3 \mu \mathrm{M}$ in the $\mathrm{LN}+$ group.
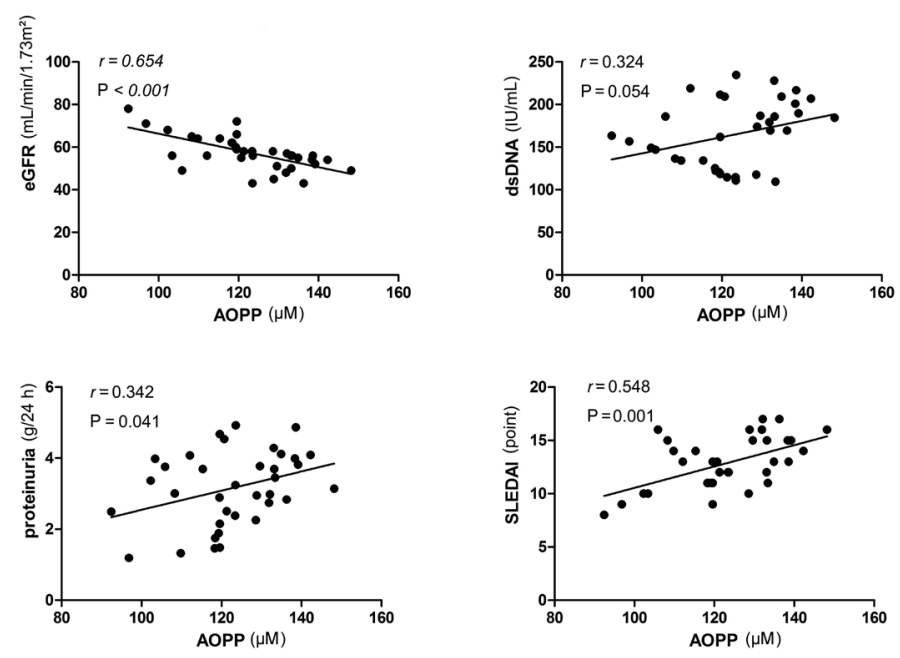

Figure 1. Correlations between clinical indices and AOPP levels in the $\mathrm{LN}+$ group.

\section{DISCUSSION}

In this study, the clinical significance of the elevated AOPP levels observed in LN patients was investigated using serum samples from SLE patients with an initial manifestation of cutaneous lupus erythematosus. The $\mathrm{LN}+$ patients had higher AOPP levels compared to those in the LN+ patients, and an elevated AOPP level was an independent risk factor for LN. Furthermore, subgroup analysis revealed that AOPP levels were correlated with proteinuria, eGFR, SLEDAI scores, and anti-dsDNA antibody levels. These findings suggest that AOPP levels may be associated with the occurrence of $\mathrm{LN}$ and could serve as a potential biomarker for predicting the severity of $\mathrm{LN}$. 
AOPP levels can be affected by numerous factors. For example, diabetes and latent infections can increase AOPP levels (Kalousova et al., 2002); therefore, patients with these conditions were excluded from the present study, which focused exclusively on SLE patients who initially presented with cutaneous lupus erythematosus.

In contrast to the present finding that AOPP levels were correlated with SLEDAI scores, Lozovoy et al. (2011) reported that plasma AOPP levels are not related to SLE activity. One possible reason for this discrepancy is that Lozovoy et al. used plasma to detect AOPPs while serum samples were used in the present study. Our recent study suggested that fibrinogen in plasma does not have the same biological features as AOPPs (Chen et al., 2011). Fibrinogen levels are elevated in SLE patients (Ames et al., 2000), while the half-life of plasma fibrinogen is decreased in SLE patients complicated with LN (Sergent et al., 1976). Therefore, fibrinogen might interfere with the applicability of AOPP levels in the evaluation of SLE activity, particularly in cases complicated by LN. Another possible reason for the discrepancy is that the study of Lozovoy et al. contained a small proportion of LN patients $(6.9 \%)$, while the present study focused exclusively on these patients.

Most SLE patients who receive treatment in the Department of Dermatology initially present with cutaneous lupus erythematosus. However, a considerable proportion of these patients do not suffer from LN (Vera-Recabarren et al., 2010). Indeed, LN can manifest during the disease course in many individuals. However, no specific biological marker has been found for LN patients in the early disease stage. The present preliminary study investigated the relationship between AOPP and LN in this population, and the findings suggest AOPP level may be a potential marker for predicting $\mathrm{LN}$.

However, this study has several limitations. First, the presence of LN as a complication was evaluated at the 6-month follow-up appointment. Therefore, the possibility of delayed LN (i.e., onset after 6 months) in the LN- group cannot be excluded. Second, because of the small sample size, it was impossible to perform a subgroup analysis of the types of skin injuries and LN. Thus, future studies with larger sample sizes and longer follow-up periods are required to corroborate the results of the present study.

In conclusion, higher serum AOPP levels are correlated with an increased risk of LN. Therefore, future studies are warranted to determine the potential clinical value of this novel biomarker.

\section{REFERENCES}

Ames PR, Alves J, Pap AF, Ramos P, et al. (2000). Fibrinogen in systemic lupus erythematosus: more than an acute phase reactant? J. Rheumatol. 27: 1190-1195.

Bombardier C, Gladman DD, Urowitz MB, Caron D, et al. (1992). Derivation of the SLEDAI. A disease activity index for lupus patients. The Committee on Prognosis Studies in SLE. Arthritis Rheum. 35: 630-640.

Capeillere-Blandin C, Gausson V, Nguyen AT, Descamps-Latscha B, et al. (2006). Respective role of uraemic toxins and myeloperoxidase in the uraemic state. Nephrol. Dial. Transplant. 21: 1555-1563.

Chen YH, Shi W, Liang XL, Liang YZ, et al. (2011). Effect of blood sample type on the measurement of advanced oxidation protein products as a biomarker of inflammation and oxidative stress in hemodialysis patients. Biomarkers 16: $129-135$.

Descamps-Latscha B, Witko-Sarsat V, Nguyen-Khoa T, Nguyen AT, et al. (2004). Early prediction of IgA nephropathy progression: proteinuria and AOPP are strong prognostic markers. Kidney Int. 66: 1606-1612.

Fismen S, Petter Rekvig O and Synnøve Mortensen E (2007). Pathogenesis of SLE Dermatitis - A Reflection of the Process in SLE Nephritis? Curr. Rheumatol. Rev. 3: 165.

Hanasand M, Omdal R, Norheim KB, Goransson LG, et al. (2012). Improved detection of advanced oxidation protein

Genetics and Molecular Research 13 (4): $9213-9219$ (2014)

CFUNPEC-RP www.funpecrp.com.br 
products in plasma. Clin. Chim. Acta 413: 901-906.

Kalousova M, Skrha J and Zima T (2002). Advanced glycation end-products and advanced oxidation protein products in patients with diabetes mellitus. Physiol. Res. 51: 597-604.

Knight JS and Kaplan MJ (2012). Lupus neutrophils: 'NET' gain in understanding lupus pathogenesis. Curr. Opin. Rheumatol. 24: 441-450.

Levey AS, Stevens LA, Schmid CH, Zhang YL, et al. (2009). A new equation to estimate glomerular filtration rate. Ann. Intern. Med. 150: 604-612.

Li HY, Hou FF, Zhang X, Chen PY, et al. (2007). Advanced oxidation protein products accelerate renal fibrosis in a remnant kidney model. J. Am. Soc. Nephrol. 18: 528-538.

Lozovoy MA, Simao AN, Panis C, Rotter MA, et al. (2011). Oxidative stress is associated with liver damage, inflammatory status, and corticosteroid therapy in patients with systemic lupus erythematosus. Lupus 20: 1250-1259.

Mok CC (2011). Epidemiology and survival of systemic lupus erythematosus in Hong Kong Chinese. Lupus 20: 767-771.

Petri M and Magder L (2004). Classification criteria for systemic lupus erythematosus: a review. Lupus 13: 829-837.

Sergent JS, Sherman RL and Al-Mondhiry H (1976). Fibrinogen catabolism in systemic lupus erythematosus. Arthritis Rheum. 19: 195-198.

Servettaz A, Guilpain P, Goulvestre C, Chereau C, et al. (2007). Radical oxygen species production induced by advanced oxidation protein products predicts clinical evolution and response to treatment in systemic sclerosis. Ann. Rheum. Dis. 66: 1202-1209.

Vera-Recabarren MA, Garcia-Carrasco M, Ramos-Casals M and Herrero C (2010). Comparative analysis of subacute cutaneous lupus erythematosus and chronic cutaneous lupus erythematosus: clinical and immunological study of 270 patients. Br. J. Dermatol. 162: 91-101.

Witko-Sarsat V, Friedlander M, Capeillere-Blandin C, Nguyen-Khoa T, et al. (1996). Advanced oxidation protein products as a novel marker of oxidative stress in uremia. Kidney Int. 49: 1304-1313.

Witko-Sarsat V, Friedlander M, Nguyen KT, Capeillere-Blandin C, et al. (1998). Advanced oxidation protein products as novel mediators of inflammation and monocyte activation in chronic renal failure. J. Immunol. 161: 2524-2532.

Yazici C, Kose K, Calis M, DemIr M, et al. (2004). Increased advanced oxidation protein products in Behcet's disease: a new activity marker? Br. J. Dermatol. 151: 105-111. 